




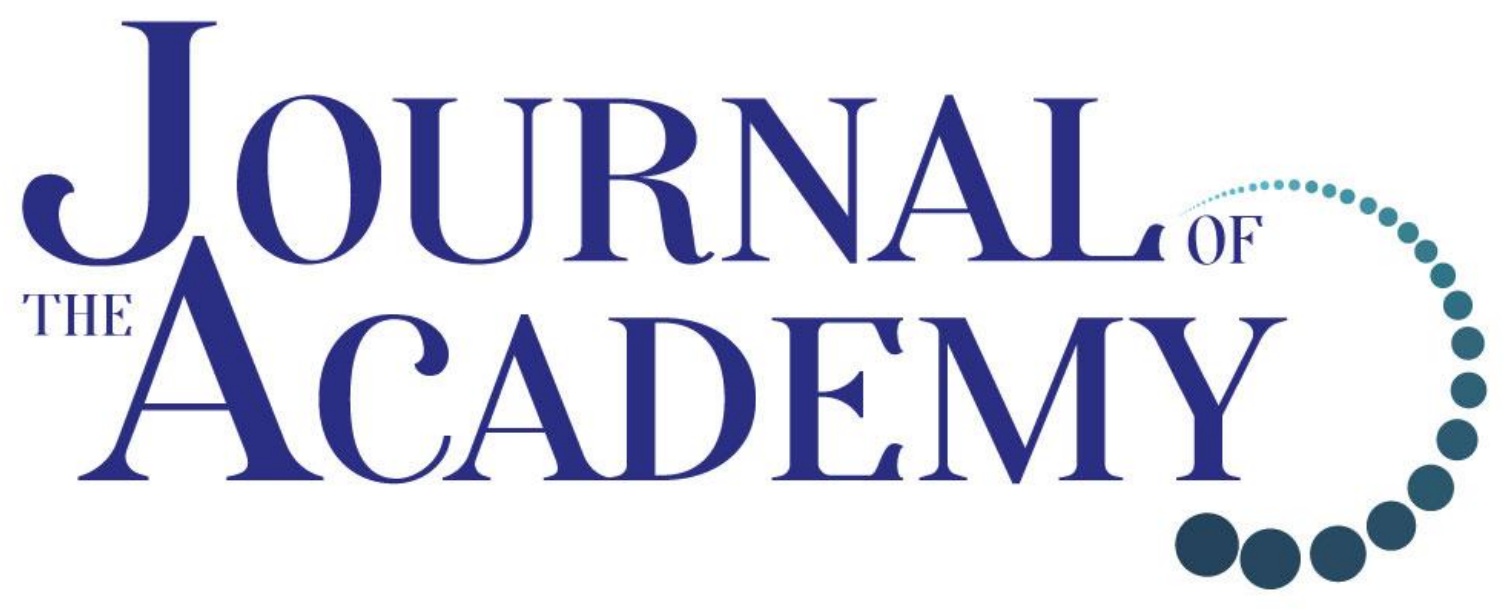

\section{Publicación Científica de la Asociación de Universidades del Perú ASUP}

Número 1

Julio-Diciembre 2019

www.journalacademy.org 
ISSN 2707-0301

\author{
Directora-Editora en Jefe \\ Ada Gallego Ruiz Conejo \\ Editor Científico \\ Oswaldo Orellana Manrique \\ Comité Científico \\ Javier Carreón Guillén \\ Universidad Nacional Autónoma de México, México \\ Martino Contu \\ Universidad de Sassari, Italia \\ Jorge Enrique Elías Caro \\ Universidad del Magdalena, Colombia \\ Roberto Escalante Semerena \\ Universidad Nacional Autónoma de México, México \\ Oscar Ortega Arango \\ Universidad Autónoma de Yucatán, México \\ Alex Veliz Burgos \\ Universidad de Los Lagos, Chile \\ Comité Editorial \\ Manuela Garau \\ Centro Studi Sea, Italia \\ José Manuel González Freire \\ Universidad de Colima, México \\ Carlos Tulio da Silva Medeiros \\ Diálogos en Mercosur, Brasil \\ Eduardo Gomes Onofre \\ Universidade Estadual da Paraíba, Brasil \\ Equipo Ejecutivo \\ Juan Carlos Norabuena Castañeda \\ Thalia Chávez Cortéz \\ Journal Academy \\ Revista Semestral Open Access
}




\title{
LA AUTONOMÍA UNIVERSITARIA COMO LEGADO DE LA REFORMA DE CÓRDOBA
}

\section{THE UNIVERSITY AUTONOMY AS A LEGACY OF CÓRDOBA}

Recibido: 22 de julio de 2018

Aceptado: 16 de junio de 2019

\author{
Henning JENSEN PENNINGTON \\ Universidad de Costa Rica, Costa Rica \\ Unión de Universidades de América Latina y el Caribe, México \\ hjensenp@gmail.com
}

\begin{abstract}
University Reform of 1918, better known as Córdoba Reform, Latin American public universities have important challenges and perspectives ahead. The majority of these challenges are focused on the defense of their principal duty legacy, which is the university autonomy, therefore, doing a critical reflection about this important heritage that has marked these institutions' development over the last 100 years, gains special relevance.
\end{abstract}

Keywords: Autonomy, Córdoba Reform, University, Latin America and Caribe, Democracy

Resumen: A cien años de la Reforma Universitaria de 1918, mejor conocida como Reforma de Córdoba, las universidades públicas latinoamericanas tienen importantes retos y perspectivas por delante. Muchos de estos desafíos se centran en la defensa de su ejercicio del legado principal de esta reforma -la autonomía universitaria-, y por ello cobra especial relevancia hacer una reflexión crítica acerca de esta importante herencia, que ha marcado el devenir de estas instituciones a lo largo de un siglo.

Palabras claves: Autonomía, Reforma de Córdoba, universidad, América Latina y el Caribe, democracia

\section{Para Citar este Artículo:}

Jensen Pennington, Henning. La autonomía universitaria como legado de la Reforma de Córdoba. Journal of Academy num 1 (2019): 13-31.

Licencia Creative Commons Atributtion Nom-Comercial 3.0 Uunported

(CC BY-NC 3.0)

Licencia Internacional

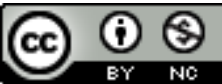




\section{Introducción}

Este ensayo es una aproximación analítica sobre la autonomía universitaria que requiere ser reflexionada a la luz del presente y desde una perspectiva que trascienda los aspectos jurídicos y de gestión universitaria, para apreciar su significación política y social, así como del espíritu que la inspiró. La autonomía universitaria es compleja y se podría afirmar que constituye la expresión democrática y crítica de una universidad y un pueblo en el devenir de una sociedad heterogénea, desigualdad y contradictoria.

En el marco del centenario de la Reforma de Córdoba, la reflexión acerca de la situación actual de las universidades latinoamericanas cobra especial relevancia. La conquista de la autonomía universitaria inauguró la posterior democratización y liberación del pensamiento de los intereses e intencionalidades del poder establecido en el Estado y la sociedad latinoamericana de la época, en la cual la universidad como institución se encontraba subordinada a diferentes vertientes de la Iglesia Católica. El acceso a la diversidad cognoscitiva y la posibilidad de cuestionar y crear conocimiento propio, contextualizado con la sociedad y la época, así como el brindarlo a la población sin restricción, inició una etapa que potencializó la conquista de nuevos derechos y el progreso de la sociedad.

Pero, la autonomía universitaria fue una conquista que requiere ser recreada permanentemente, según se modifican las circunstancias, fenómenos, conocimientos y las formas que adopta el poder en una sociedad desigual y contradictoria. Aspectos que no siempre han sido contemplados en el devenir cotidiano universitario, razón por la cual se postula que silenciosa y acríticamente se pierde autonomía por agresiones o sumisión externa y por la relativa indiferencia interna, no necesariamente consciente ni intencionada, por parte de la colectividad universitaria. Una entidad de educación superior pública sin autonomía destruye su libertad y los vínculos con su sociedad y época. Los retos que se enfrentan son varios, pero sumados a la experiencia que ya han tenido diversas instituciones en la región, se presentan al final de este ensayo algunas reflexiones sobre estos desafíos y la necesidad de actuar sobre ellos.

\section{Reflexiones sobre la universidad de Humboldt a la de nuestros días}

En los años setenta del siglo pasado, la Universidad de Costa Rica (UCR) pretendió materializar y hacer compatibles dos ideas de universidad: por un lado, la idea humboldtiana de la universidad clásica alemana $y$, por otro, la idea más propiamente latinoamericana de una universidad comprometida con las aspiraciones de su gente.

En este segundo aspecto, la UCR incorporó en su quehacer, de una manera más vigorosa que antes, el legado de la reforma de Córdoba y los debates políticos de la época, marcados profundamente por la teoría de la dependencia y los movimientos de liberación nacional en diferentes países latinoamericanos. Empezaré este ensayo con una reflexión sobre estos temas. ${ }^{1}$

Ya Humboldt y Schleiermacher habían postulado la inseparabilidad de docencia e investigación en la Alemania del siglo antepasado; para ellos, el sujeto del saber no era el pueblo, sino el espíritu especulativo. Detrás de esta posición filosófica, se encontraba el proyecto de liberar la ciencia y el conocimiento de la injerencia del Estado.

\footnotetext{
${ }^{1}$ Estos temas han sido discutidos en textos ya clásicos de Jürgen Habermas y otros autores, en los cuales me baso ampliamente.
} 
Esa idea aspiraba a la articulación concreta de dos aspectos: (1) La institucionalización de la ciencia moderna, entonces ya liberada de la tutela de la religión y la Iglesia, sin que su autonomía llegase a ser amenazada por la autoridad estatal, de la cual dependía económicamente, ni por la sociedad burguesa, la cual podía obtener beneficios de los resultados del trabajo científico. (2) Serviría a los intereses del Estado garantizarle a la universidad un espacio de ilimitada libertad, en virtud de las prósperas consecuencias que para la vida de la nación acarrearía la ciencia institucionalizada, promotora de la unidad nacional y de la cultura moral.

Estos dos aspectos le imprimieron su sello característico a la tradición universitaria alemana y de ahí se explican los siguientes rasgos: (1) la relación afirmativa con el Estado de una ciencia universitaria que se concebía a sí misma como "apolítica»; (2) la actitud defensiva de la universidad ante la práctica profesional, cuyas exigencias podían amenazar la unidad de docencia e investigación; (3) la posición central de las facultades filosóficas dentro de la universidad y la relevancia que con ello se le otorgó a la ciencia para la cultura y la sociedad. Finalmente, (4) a la unidad de docencia e investigación debía corresponder especularmente la unidad de docentes y estudiantes, basada en una relación de cooperación y complementariedad. Humboldt decía que el profesor no se encontraba allí para servir al estudiante, ni éste para servir a aquél; sino que ambos estaban allí para servir a la ciencia.

El problema de la idea humboldtiana de universidad es que fue rebasada por sus propios éxitos, a saber por el desarrollo de las ciencias naturales experimentales y la evolución jerárquica de los institutos de investigación; por la imposibilidad de anticipar intra muros, en el microcosmos universitario, una sociedad de personas libres e iguales; por la conversión de la ciencia en una de las más importantes fuerzas productivas de la sociedad industrial y por la destrucción de las imágenes unitarias y metafísicas del mundo por las ciencias empíricas y su ideal de racionalidad metódica.

Como sabemos, la universidad alemana y los resultados de la investigación en ella realizada nutrieron de manera incalculable el desarrollo de la industria y con ello estalló en pedazos la docencia investigativa autorreferente de la «torre de marfil».

Pero observemos también el lado positivo de la universidad humboldtiana: en tanto que idea e ideología a la vez, contribuyó al esplendor y al éxito internacionalmente incomparable de la universidad alemana en el siglo XIX. La ideología de los mandarines alemanes promovió una intensa autoconsciencia corporativa en el seno de la universidad, atrajo la promoción del Estado y obtuvo un gran reconocimiento por parte de la sociedad global. Finalmente, en algunos momentos históricos, aunque, como es evidente, no en todos ellos, el contenido utópico de la idea de universidad ha mantenido su potencial crítico ante sí misma y la sociedad.

Al pensar en la historia del proceso de modernización de las sociedades occidentales, viene a nuestra mente la imagen célebre de Max Weber sobre el desencantamiento del mundo. Esta idea hace referencia a la constitución de una visión del mundo libre de representaciones mágicas. El desencantamiento, si seguimos a este clásico de la teoría social, ha significado, entre otras cosas, la instauración de un tipo de racionalidad que gobierna tanto la actividad productiva material, como el derecho y el poder burocrático.

Hace ya muchos años, Herbert Marcuse criticaba este concepto de racionalidad y demostraba cómo él exige un tipo de acción que implica el ejercicio de poder sobre la naturaleza, la sociedad y los seres humanos. La racionalidad propia de las sociedades modernas no es la racionalidad en sí, decía Marcuse, sino la imposición de una forma determinada de dominación política que sustrae de la reflexión colectiva el proceso de elección de estrategias y la aplicación de tecnologías. 
El proceso de racionalización hace del éxito y del control, que se derivan de la aplicación adecuada de reglas técnicas, la medida de casi todas las cosas y, a la vez, el fundamento de legitimación de las formas de organización social. Lo que parece técnicamente posible, se presenta como lo socialmente deseable.

Toda esta racionalización del mundo ha afectado el desarrollo de la ciencia y la tecnología, la organización y aplicación del conocimiento, y así también ha transformado el concepto mismo de formación educativa y finalmente la idea y la estructura de la universidad; ha afectado, en suma, todas las esferas sociales. Sabemos que tanto la práctica de la medicina, como la administración de empresas, la manipulación del comportamiento electoral, la inducción al consumo y la organización del tiempo libre, para mencionar solamente algunos ejemplos, son posibles en virtud de que la aplicación del saber científico ha adquirido la forma de una disposición técnica sobre procesos objetualizados. La técnica posibilita tanto la dominación de la naturaleza como el ejercicio de poder sobre el comportamiento humano.

La formación universitaria ha cambiado también en un sentido esencial: ahora la ciencia se encuentra estrechamente acoplada con los procesos de producción y administración; es decir, existe una imbricación entre la enseñanza, el aprendizaje, la investigación y aplicación técnica del conocimiento. La educación universitaria ha de trasmitir el saber científico y las reglas de su transferencia técnica.

Esta proclividad de la educación superior hacia el pragmatismo no fue siempre tan unilateral como ahora. Antes se decía que la ciencia formaba a la persona, que la persona adquiría, a lo largo de su formación universitaria, una conciencia reflexiva que llevaría a la capacidad de interpretar el acervo científico, para así descubrir las preguntas relevantes para asegurar la buena convivencia entre los seres humanos ... y contribuir a responderlas.

Schelling decía que "... sólo las ideas le otorgan a la acción energía y relevancia ética.» Con ello quería decir que el conocimiento científico debía imprimirle su forma a la vida, pero a su vez debía siempre ser orientado por principios éticos y morales, en sentido amplio; que la ciencia, en fin, debía implicar el cultivo de una conciencia ilustrada que contribuyese a la solución de los problemas que afectan a la vida en sociedad, pero no en el sentido de soluciones meramente técnicas, sino en el de aquellas que ayudan a ampliar el horizonte de la libertad y la justicia. Conocimiento científico, entonces, como forjador de los espacios de utopía.

Lamentablemente, a las ciencias experimentales estrictas ya les es ajena esta actitud ilustrada, así como les es a las ciencias sociales que siguen su modelo. Entre nosotros se impone igualmente cada vez más la idea de que la vinculación entre universidad y sociedad ha de transitar por el sendero de la transferencia de tecnología, entendiéndose por esto el acoplamiento entre conocimiento, producción y administración. Es cierto que la universidad no puede desatender este asunto, como tampoco antes lo ha descuidado del todo, pero la pregunta sustancial acerca de cómo este tipo de acoplamiento ha de contribuir al desarrollo de la justicia y la libertad, no encuentra todavía respuesta, y menos la encontrará si este tema no se convierte en objeto de reflexión de la misma ciencia.

\section{La Reforma de Córdoba como detonante para la Autonomía Universitaria en América Latina}

La autonomía universitaria se aproxima a cumplir una centuria de haberse construido colectivamente en Córdoba, a la vez que mostraba en su tiempo la existencia de las condiciones para su constitución latinoamericana. Desde su origen hasta nuestros días son muchos los escritos efectuados sobre la autonomía; sin embargo, no se agota su importancia y significación para el 
desarrollo universitario y social nacional y mundial. Las distintas contradicciones con los poderes prevalecientes en una sociedad inequitativa, que nos recuerdan en otros contextos sus luchas originarias, han contribuido a deconstruir y recrear inéditamente sus contenidos en cada tiempoespacio social.

La autonomía universitaria fue una conquista democrática estudiantil en 1918, pero no estuvo aislada de un contexto social que aspiraba y reclamaba libertad e igualdad, como tampoco lo fueron los movimientos estudiantiles de 1968 en París, Varsovia, Roma, Berlín, Berkeley, Tokio y México, a los que Fernand Braudel lo apreciaba conformando una "revolución cultural"; una "efervescencia revolucionaria" según Claude Lefort, o la inversión de la hegemonía cultural dominante, desde la perspectiva de Immanuel Wallerstein. Fueron movimientos que revolucionaron la educación al servicio político e ideológico del poder, para convertirla en una institución autónoma al servicio de todos los sectores sociales. Los movimientos estudiantiles inauguraron lo público en el ámbito de la educación superior en América Latina transformando las universidades en entidades autónomas en su gestión y en la difusión y generación de conocimiento científico. La conquista de la autonomía universitaria fue una gesta democrática para las universidades y las sociedades.

La universidad lidió, en su pasado prerreformista, con las doctrinas religiosas que le imponían una actuación dogmática, como también lo hizo contra los poderes privados y autocráticos establecidos en la sociedad y el Estado. Las reformas universitarias de 1918 removieron las bases aristocráticas de las universidades e inauguraron la posibilidad del florecimiento de las universidades públicas con libertad de pensamiento y compromiso social; sin embargo, los embates liberales de las últimas cuatro décadas han debilitado su actuación pública. En algunos contextos han perdido total autonomía con respecto del saber y del poder político y económico, en otros casos se han independizado de las necesidades públicas y se han coludido ideológicamente con el poder económico y político autocrático.

Los movimientos estudiantiles crearon, a través de la constitución de la universidad pública, las bases democráticas en momentos que las sociedades no erradicaban el espíritu elitista y aristocrático de la época. El movimiento de Córdoba declaraba su empeño por la libertad e igualdad por el logro de la participación estudiantil en la gestión universitaria, por la extensión cultural de los universitarios, por la difusión del pensamiento nacional, entre otros aspectos. Dicho movimiento no fue exclusivamente argentino; fue también un movimiento de latinoamericanos. Su actuación ha tenido una vigencia a través de los tiempos porque inauguró la posibilidad de pensar y actuar libre y democráticamente en un espacio social sensible y vital para el progreso.

La conquista de la autonomía universitaria convulsionó las sociedades latinoamericanas y creó las bases de una nueva época. Se había conquistado un derecho y se estaba ante un escenario distinto en las relaciones entre la educación superior y la sociedad. Se había gestado la ruptura del poder autocrático con el pensamiento. La autonomía reveló el antagonismo en el sistema social imperante, que no se resolvió con su formalización jurídica, pero evidenció y visibilizó la necesidad de limitar tendencias, tensiones o agresiones contra la universidad.

\section{La relación entre Universidad, Estado y Sociedad}

La pretensión por subordinar la autonomía no termina con su reconocimiento constitucional; renovar creativamente la libertad en cada tiempo-espacio es una necesidad en la heterogeneidad de las relaciones sociales. La democracia creada socialmente en las universidades, constituyeron procesos inéditos, sin los cuales no es posible la autonomía. 
La universidad no ha sido nunca, en realidad, torre de marfil; su definición como tal es mera ideología y negación del hecho que el quehacer universitario no responde a supuestas obligatoriedades inmanentes del conocimiento, así como tampoco es cierto que el progreso técnico se encuentra totalmente libre de decisiones precientíficas.

$\mathrm{Si}$ el estudio universitario implica la transformación del conocimiento en obras, como lo expresaban los teóricos de los siglos XVIII y XIX, entonces es ahora urgente hacer de las reglas, a que está sometida esa transformación, un objeto de discusión por parte de una ciencia orientada hacia el interés general, con el propósito de ilustrar a la esfera pública sobre las consecuencias prácticas del desarrollo científico y tecnológico.

No está de más recordar aquí aquella frase de Theodor W. Adorno, tan enigmática como cierta, que dice: "el progreso acontecerá, en el momento en que termine.» Quería Adorno decir con esto que el progreso material ha acarreado grandes beneficios para todos, pero a la vez le ha abierto las puertas a la inhumanidad. El progreso se iniciaría, entonces, en el momento en que pierda la falsa apariencia de una automática obligatoriedad y sus condiciones sean reflexionadas a la luz de los intereses públicos. Entre muchas otras cosas, todo esto implica el desarrollo inagotable de la responsabilidad social.

La relación entre universidad, Estado y sociedad se encuentra sometida a un cambio que posiblemente sea radical. En este proceso de transformación, la universidad (y sobre todo la pública) debe lograr el desarrollo de una voluntad política que le permita intervenir en la elucidación de los asuntos que incumben a la comunidad entera. Pero esto sólo puede alcanzarse mediante la preservación de la autonomía de la universidad, ya que únicamente en estas condiciones de libertad pueden descubrirse las implicaciones sociales del conocimiento y puede desplegarse la autorreflexión de una ciencia que ahora ya no es posible concebir como apolítica.

Existe también un factor de naturaleza sociológica que no deseo pasar por alto. La formación universitaria ha conducido al surgimiento de estratos sociales que consolida las diferencias en nuestra sociedad, estratos sociales que ahora defienden sus privilegios y atacan el sistema universitario estatal y público; es decir, la universidad que contribuyó a formar ese mismo estrato social, pero que ahora. En otras palabras, el sistema universitario público no ha podido asegurarse la lealtad de muchos de sus graduados, porque ha sido parte de los mecanismos de movilidad social que han creado ese estrato que más se ha beneficiado directamente del Estado social y que ahora, en situación de crisis, entra en una alianza defensiva con el sector llamado productivo y algunos sectores políticos en contra de los desposeídos y marginados. Y estos - los excluidos de los beneficios sociales, culturales y económicos - expresan sus ahogados anhelos mediante alianzas transitorias con los agentes políticos que prometan cumplir sus ilusiones, aunque todo ello desemboque en un mayor desencanto. Todo ello significa que las alianzas tradicionales de la universidad se han debilitado y todavía no hay respuesta definitiva a la pregunta de si esta institución cultural podrá asegurarse el apoyo de nuevos sectores de la población.

Existe otro factor meritorio de toda atención en el presente contexto: la globalización económica. La universidad pública constituye, en muchos países latinoamericanos, un núcleo generador de identidad cultural, la cual, a su vez, puede representar un quizá débil, pero importante obstáculo para la internacionalización de los mercados. De esta situación se deriva una política neutralizadora de las idiosincrasias nacionales, pero también un nuevo reto para la universidad y, en especial, para las ciencias sociales: contrarrestar la repercusión cultural de la globalización, a sabiendas de que la solidaridad entre los seres humanos se basa, entre otras cosas, en la posibilidad de descubrirse como partícipes de una misma identidad cultural. 


\section{La Universidad Pública y su papel hacia el Estado, la cultura y la ciudadanía}

La idea de universidad continúa siendo, pues, un elemento correctivo. En esta idea está implícito un proceso de aprendizaje, de producción y acumulación de conocimientos, que se lleva a cabo mediante un esfuerzo cooperativo de argumentación. Si la sociedad global obtiene de la universidad algo ejemplar para toda ella, consiste esa contribución precisamente en la vitalidad, en la fuerza impulsora y productiva que nace de la disputa libre y pública de ideas y argumentos. Bien podríamos afirmar que a la idea de universidad le es inherente la forma ideal del discurso autónomo y del pensamiento sorpresivo. En todo ello quiere expresarse una utopía que todavía cree en la posibilidad de la libertad de los seres humanos y de los pueblos.

Podemos afirmar que el grado de desarrollo de una sociedad se mide en el potencial de aprendizaje estructuralmente tolerado y fomentado, en la capacidad de admitir argumentaciones razonadas y de fundamentar consensualmente la administración técnica y política.

Estos procesos de aprendizaje colectivo dependen del grado de realización de la idea de democracia y de los espacios permitidos de participación política de los ciudadanos. Quienes vivimos a principios del siglo veintiuno lamentamos constatar que la idea de democracia se encuentra ya lejos de su digna definición como poder del pueblo, para el pueblo y por el pueblo; sentimos ser testigos de que la democracia se ha tornado sinónimo de un conjunto de reglas de un juego en que algunos individuos acceden a la facultad de decidir gracias a la obtención de los votos de un pueblo.

No obstante, debemos recordar que la democracia debe ser irreductible a un método para alcanzar poder político y administrativo; en su más íntima esencia se encuentra el anhelo de autodeterminación del pueblo: sólo cuando ésta se haya realizado plenamente, se habrá puesto en práctica también la democracia. Pero esta idea se ve sorprendida por la resistencia que a ella opone la concepción de democracia como tecnología social, ahora tristemente célebre y eficientemente ejecutada merced al cinismo de una generación que se cree a sí misma postideológica, pero que, en realidad, sólo es denegatoria de la consciencia histórica.

En esta época -que algunos llaman la era de la angustia-, la enseñanza superior es sometida crecientemente a los criterios de performatividad propios de la esfera de la producción. Este concepto de performatividad, resultante de una sociedad que ha encontrado en la banda transportadora su más elocuente metáfora, implica un abandono de las tareas universitarias que aspiraban a la formación y la difusión de un modelo general de vida, ligado en mucho a la idea de la emancipación colectiva. Como lo manifiesta Jean-Francois Lyotard, "las universidades y las instituciones de enseñanza superior son de ahora en adelante solicitadas para que fuercen sus competencias, y no sus ideas...».

No deja de ser una curiosidad de nuestros tiempos que el predominio de la razón instrumental sobre la razón práctica estimule la formación de un tipo de ser humano que, en la psicología clínica y en la social, fácilmente adquiere los rasgos de una patología caracterológica. En uno de los más reputados proyectos de investigación social de la historia, los estudios sobre la personalidad autoritaria de Theodor W. Adorno, se estableció la categoría del «carácter manipulador», el cual se caracteriza por su furor organizador, su incapacidad de hacer experiencias humanas inmediatas, una especie de carencia afectiva y sobrevalorado realismo, el culto a la eficiencia y una a veces delirante Realpolitik. En suma, es el tipo humano de una consciencia reificada para quien los otros constituyen sólo una masa amorfa. 
Poco antes de la Segunda Guerra Mundial, el poeta francés Paul Valéry decía que la inhumanidad tenía un gran futuro. No sospechaba quizá el grado de extrema barbarie con que la historia posterior inmediata confirmaría sus temores. A todo ello se ha opuesto desde siempre la idea de universidad; esa idea que se resiste a las imposiciones de un «taylorismo espiritual», la idea en que late todavía una utopía de libertad que desea restarle a la inhumanidad la posibilidad de instalarse en el mundo sin ser cuestionada.

En estas reflexiones, lo que he querido transmitir es que la universidad pública crea Estado, forja cultura y templa las fuerzas de la ciudadanía. Esta función de la universidad pública no se aviene bien con las tendencias de imperio del mercado; más bien, constituye un foco de resistencia que, desde la perspectiva de la economía liberal, debe ser debilitado

Boaventura de Souza habla de internacionalidad solidaria como principio para reafirmar esta funciones tradicionales de la universidad pública en un contexto de globalización económica y socavamiento de las identidades nacionales. Este es un principio que ha de actuar como factor rectificador de relaciones asimétricas y carentes de complementariedad en las agendas académicas de las universidades latinoamericanas y las del mundo desarrollado. Diferentes evaluaciones se han llevado a cabo para determinar el grado de éxito de estos esfuerzos.

En efecto, esa asimetría se debe sin duda a factores económicos y políticos, mientras que la ausencia de complementariedad obedece al hecho de que los países desarrollados y los países en desarrollo tienen diferentes prioridades académicas, especialmente en investigación. Un ejemplo de ello se encuentra en la investigación médica: los europeos, por ejemplo, tienden a especializarse en pocas enfermedades tropicales, mientras que los científicos latinoamericanos, en atención a las demandas de sus propias poblaciones, le dan mayor importancia a la medicina preventiva, a problemas de salud relacionados con el ambiente y con los regímenes alimentarios. Tanto la salud pública como la administración de servicios de salud son de gran importancia para los países en desarrollo. En otros campos, Europa y el Tercer Mundo muestran diferentes intereses, por ejemplo en la investigación agrícola.

Es oportuno recordar que, en la revista Science del 7 de marzo del 2003, Kofi Annan decía: "Los países en desarrollo gastan mucho menos que el uno por ciento de su producto interno bruto en investigación científica, mientras que los países ricos dedican entre el dos y el tres por ciento. La proporción de científicos en los países en desarrollo es de 10 a 30 veces menor que en los países desarrollados. El 95\% de innovaciones científicas se lleva a cabo en países donde vive un quinta parte de la población mundial. Y la mayoría de esa ciencia - en el ámbito de la salud, por ejemplo - desdeña los problemas que afectan a la mayoría de la población mundial" Este desequilibrio, continúa Annan, "acentúa la desigualdad entre países avanzados y en desarrollo y crea dificultades sociales y económicas nacionales e internacionales."

No cabe duda de que existe una división de trabajo científico entre los países desarrollados y en desarrollo. En términos generales, los científicos de países en desarrollo participan activamente en las fases operativas de los proyectos conjuntos de investigación, pero no están igualmente involucrados en otras fases, tales como la conceptualización teórica y metodológica de los proyectos, el análisis y la discusión de los resultados y la redacción de las publicaciones de los resultados correspondientes. Los científicos de los países en desarrollo tienden a estar más involucrados en los procesos de recolección de datos, lo cual constituye una participación muy limitada que no se traduce necesariamente en una potenciación de las capacidades científicas endógenas. Una consecuencia de ello es que los científicos de países desarrollados tienen a publicar mucho más y a participar en eventos científicos con mucha mayor frecuencia que sus socios de países en desarrollo. A pesar de que los nombres de los científicos de países en 
desarrollo aparezcan en la lista de autores de las publicaciones correspondientes, ello no es expresión necesaria de una participación simétrica ni de formación de masa crítica. Por tanto, en los proyectos internacionales debemos aplicar una ética de la cooperación que nos garantice una participación sustantiva, más allá de una simple maquila científica.

\section{Las Universidades latinoamericanas y el desarrollo de sus principios universales}

Las universidades nunca fueron espacios aislados ni descontextualizados de sus naciones y regiones, aun cuando no necesariamente fueron conscientes de sus determinaciones sociales, históricas, ni sobre sus responsabilidades en sus sociedades. La autonomía universitaria de la Universidad Pública no es la concreción privada que imaginan algunos y que ilusamente la comparan con las empresas lucrativas que tienen en la educación el medio para lograrlo.

Es oportuno detenerme a explicar que, regularmente, la universidad pública es estatal, porque sus bienes y financiamiento son del Estado, así como es creada para cumplir funciones de formación superior al conjunto de la sociedad y contribuir con su desarrollo. Sin embargo, lo público no se circunscribe al quehacer estatal, dado que el presente liberal, muchas acciones gubernamentales no están inspiradas en la representación y amparo del interés general de la sociedad, ni es la ciudadanía, sin distingos ni exclusión, el conjunto de beneficiarios. Aún cuando lo privado es consustancial a lo público, la imposición privada como equivalente de lo ciudadano y nacional es una destrucción de lo público. La instauración de lo público no es inherente a todas las sociedades y épocas, lo es en aquellas que constituyen sociedades de derecho igualitario y democrático. Las universidades públicas deberán tener en lo común a todos y en su sociedad, el ámbito de su quehacer y preocupación cotidiana. Esto es, no es lo estatal lo característico en el devenir de la universidad pública, sino su compromiso y vínculo con lo colectivo y nacional en un mundo integrado. Este atributo es el sustento de su autonomía, no es la condición estatal lo que define su actuación cotidiana.

Las luchas y reivindicaciones universitarias de Córdoba traspasaban las efímeras fronteras nacionales para convertirse esencialmente en procesos sociales, no exclusivamente reivindicativos. Ellas abrigaban transformaciones radicales en sus sociedades.

La reforma universitaria lograba la autonomía o la libertad de pensamiento y al socializarlo potencializaba el progreso y la democracia de las sociedades. La renuncia al conocimiento exclusivo y excluyente, brindaba acceso a la educación a otros segmentos y clases sociales, a la vez que se sustituía el pensamiento arcaico, que era el fundamento que perpetuaba la dominación política y religiosa.

La democracia universitaria, así como el progreso, desarrollo y bienestar, son expresiones que tienen una extraordinaria aceptación y difícilmente se explícita su oposición, porque pone en evidencia un pensamiento y una acción contra la libertad de pensamiento y el progreso. La libertad del conocimiento diversifica y transforma interpretaciones y explicaciones de las realidades y con ello modifica la conciencia de los individuos y los escenarios de actuación colectiva para crear nuevos destinos compartidos.

Habría que admitir que las tiranías, en el presente y pasado de militares o civiles, han justificado sojuzgar la libertad de pensamiento en el nombre de la democracia, el orden y el statu quo. Argumentar o exigir democracia para el ejercicio de la dictadura es una vieja práctica empleada por las más sanguinarias tiranías que ha conocido la humanidad. Hitler la demandaba, como muchos tiranos en América Latina y el mundo, en el pasado y el presente, se autodefinen como democráticos . 
La democracia es una conquista social; es la liberación de la población de toda forma de decisión, opresión y explotación privada que atente contra la vida, voluntad e interés general de la sociedad. La autodeterminación colectiva es la manifestación democrática de los individuos en la sociedad.

La democracia es una dinámica relación social, provista de la significación que le otorga la vida compartida en cada tiempo y espacio, constituyendo una propuesta y acción crítica y transformadora de las relaciones sociales y políticas dominantes. El momento democrático es el espacio social donde se conquistan derechos en una sociedad desigual e inequitativa, en una época particular. No existe democracia del statu quo, ni las conquistas son equivalentes en diferentes contextos sociales e históricos. La democracia es un proceso histórico moral, inédito para las colectividades y sociedades que lograron la construcción de lo común y lo público en los espacios globales compartidos.

La constitución de lo público, de lo que es común a todos, fue un evento democrático de las masas que conmovieron sociedades e impusieron pautas de actuación a los Estados, que debían actuar en razón de concretas problemáticas, intereses y anhelos de la pluralidad ciudadana, pero paradójicamente también para el sustento en la subsunción real del trabajo por el capital que consume o destruye democracia en el proceso organizativo de la producción. La libertad y democracia no son una creación ni dádiva estatal, aunque son una construcción colectiva que debe crear las condiciones políticas que posibiliten la reproducción económica y la formalidad de las relaciones igualitarias en la sociedad. De este modo, la constitución del derecho igualitario en las sociedades otorgaba legitimidad política a la valorización del capital e inauguraba la individualización y la supeditación del trabajo y la expansión de relaciones predominantes, pero a la vez contradictoriamente, el poder en las relaciones privadas destruía libertad y democracia en el proceso de valorización del capital, creando un círculo perverso de dominio político.

Los movimientos sociales dieron consistencia, viabilidad e integración a un sistema que requería de individuos libres e iguales. Los derechos individuales fueron conquistas democráticas que revolucionaron a las sociedades y los Estados atrasados, donde las facultades de unos pocos, contrastaban con la carencia de derechos de muchos. La libertad e igualdad son condición fundamental de los derechos individuales en una sociedad desigual, pero también se constituyen en el medio para la expansión de la organicidad, fortalecimiento y expansión capitalista. El equilibrio construido democráticamente es inestable, ante la cosificación de las relaciones sociales que expande relaciones privadas de poder.

La autonomía universitaria es evocada contemporáneamente en Costa Rica, como en otros países de América Latina, ante muchos intentos por subordinar el quehacer universitario a formas políticas predominantes o emergentes, tanto desde dentro como fuera del claustro universitario.

La autonomía universitaria es el derecho al ejercicio de la libertad de pensamiento en el quehacer universitario cotidiano. Contrario a lo que algunos detractores de la universidad pública afirman, la autonomía universitaria no es la inmunidad ni impunidad de actuación, y tampoco es una entidad independiente del Estado. La libertad de pensamiento es la manifestación de mujeres y hombres libres e iguales: atentar contra la posibilidad de expresar las reflexiones, interpretaciones y criterios personales y colectivos, es quizás la forma más infame de contravenir a los derechos de los individuos y las sociedades.

La autonomía universitaria o la conquista de libertad de pensamiento en el espacio de la universidad, es el reconocimiento de la condición necesaria para que la entidad difunda y cree conocimiento científico en una sociedad y una época particular. La autonomía universitaria es la condición de un pueblo libre y el derecho ciudadano a la educación que se corresponda con el 
estado del conocimiento, para conocer, comprender e interpretar realidades concretas, pero también para la ubicuidad y la identidad comprometida con los procesos que ocurren en las sociedades. Es esta la característica por la cual se considera a la educación como una condición necesaria para el desarrollo, pero deberá ser una educación pertinente y consistente con la actualidad cognoscitiva, la sociedad y la época, así como con la prospectiva común de desarrollo social. De otro modo, solo será una entidad reproductora de ideología y conocimientos funcionales a los poderes predominantes y privativos.

La autonomía de una universidad trasciende a la defensa de un espacio físico, sin embargo lo defendemos porque es el ámbito para el ejercicio académico libre y porque la transgresión y ocupación del espacio universitario ha sido el medio para violentar la libertad y derecho al conocimiento y al pensamiento crítico. Me permito rescatar que la criticidad es una característica de todo conocimiento científico, dado que no existen realidades iguales ni estáticas, y porque no existen explicaciones idénticas para fenómenos en espacios y tiempos distintos. La ciencia en su desarrollo deconstruye conocimientos para ser sustituidos por otros con mayor capacidad explicativa. Del mismo modo, una universidad que no promueva la criticidad del pensamiento será una simple institución que impone criterios y conceptos, reproduciéndolos sin cuidado de su profundidad o pertinencia, pero especialmente, haciendo que quienes forman parte de ese proceso formativo consideren que se trata de conocimientos acabados en sí mismos, sobre los cuales se puede basar toda una carrera profesional.

\section{Los retos de las Universidades latinoamericanas por una región más equitativa}

Los retos de las universidades latinoamericanas son muchos y de muy diversas índoles. Tras conocer en primera persona la experiencia de las instituciones que forman parte de la Unión de Universidades de América Latina y el Caribe (UDUAL), me detendré en algunas de las áreas que considero prioritarias para promover un papel activo de nuestras universidades en la construcción de una región más equitativa, justa y orientada hacia el desarrollo sostenible, como un actor más dentro del complejo tejido social en el cual cada persona y cada institución tiene importantes responsbilidades para con su propio progreso.

En primer lugar, la autonomía es una necesidad y condición para una formación universitaria de calidad. La educación no es una mercancía; no es un objeto que deba acumularse o apropiarse para beneficio privado. Además de ser un derecho y un bien, es una necesidad social y cultural, una facultad para conocer las realidades y actuar consecuentemente en cada contexto. En este sentido, la autonomía trasciende el espacio universitario, para estar vinculada con el derecho ciudadano y el desarrollo. Contar con un entorno social que comprenda esta primordial razón de ser de la educación, defenderá y promoverá más y mejores espacios de formación formal y no formal, dentro de los cuales las universidades podrán tener un papel predominante. Reconocer que es allí en donde se genera el pensamiento, la actitud crítica y la sensibilidad de las y los futuros profesionales de un país, permitirá que los recursos que sean destinados a las universidades públicas sean más pertinentes para las necesidades que implican la docencia, la investigación y la acción social o extensión.

Cada uno de estos tres pilares universitarios retribuye a la sociedad con profesionales formados a la luz del humanismo y el razonamiento crítico, aspectos necesarios para ejercer cualquier profesión con ética y con consciencia ciudadana; retribuye también con nuevos conocimientos aplicables a las realidades locales y regionales en nuestra América Latina, con necesidades tan particulares pero que a la vez podrían extrapolarse para aprender de las experiencias de otras 
regiones; y retribuye directamente hacia las comunidades con las cuales ha estrechado lazos de colaboración mutua. Pero, por supuesto, el otorgamiento de recursos siempre deberá ser sujeto de una constante supervisión, no solo por parte de las autoridades nacionales o cooperantes, sino también por la propia ciudadanía. Este, quizá, es a fin de cuentas un reto común con la sociedad, al hacerle entender que las universidades son su patrimonio, que deberá cuidar y proteger, y a la vez exigirle los resultados que espera para su propio progreso.

Por otra parte, reitero que una universidad no puede concebirse como aislada de la sociedad que la contiene, y que le da vida. Una de las responsabilidades de la educación superior es ser promotora de las acciones que beneficien a la sociedad, para un desarrollo continuo y parejo que favorezca a la población más vulnerable; en esta línea, el enfoque de cada uno de los pilares sustantivos que componen el quehacer universitario debe siempre tener en consideración que la nuestra es una sociedad desigual, y que por lo tanto requiere de una visión sensible hacia las inequidades que la agobian. Esto no quiere decir privilegiar o discriminar bajo ningún contexto; al contrario, significa brindar las mismas oportunidades a quienes de otro modo no las tendrían disponibles.

Particularmente me refiero a las oportunidades de acceso a la educación superior, que tienen una larga trayectoria de obstáculos si se considera las oportunidades que cada niño y niña de nuestra región tiene para acceder a la educación general básica, o primaria, y continuar a la secundaria. Quienes alcanzan la educación superior, representan una pequeña parte de quienes iniciaron juntos sus primeros pasos formativos en la escuela, y esto genera una terrible desigualdad entre pares de una misma comunidad. Posibilidades económicas, necesidades familiares o incluso las perspectivas de movilidad de una comunidad, son solo algunos de los factores que pueden incidir en que un niño o una niña no alcancen a pisar los umbrales de una institución de educación superior. Sin embargo, las universidades pueden y deben establecer mecanismos para promover que sean más personas quienes puedan acceder a esta educación, entendiéndola como el principal factor de movilidad social al que podría aspirar una persona, por sus propios méritos.

Dotar de un sistema de becas adecuado y robusto, que cubra y alcance para sostener a un joven en la educación superior, y que a la vez permita dar un respiro económico a su familia al no tener que velar por ese estudiante, es parte de las respuesas que una universidad puede brindar. Sin embargo, la institucionalidad nacional y la propia ciudadanía deben dar un paso al frente para permitir que las universidades tengan y dispongan de los recursos necesarios para permitir esto. Las becas no sean el único sistema para permitir el ingreso y mantenimiento de las y los estudiantes universitarios en condiciones de vulnerabilidad, pero quizá son el motor que brinda movimiento para que cada vez más proyectos -incluso generados bajo esa propia sensibilidadpuedan alcanzar a las comunidades más vulnerables.

Seguidamente debo mencionar el aspecto tecnológico. La tecnología es transversal a todas las áreas del conocimiento; las universidades públicas deben apropiarse también de los procesos tecnológicos que actualmente permean múltiples actividades cotidianas. No hacerlo sería una sentencia para la efectividad de estas instituciones, que no solo requieren mantenerse a la vanguardia para ofrecer mejores servicios, sino que deberían estar en capacidad de fomentar su uso y consecuente apropiación por parte de la comunidad universitaria.

Las innovaciones tecnológicas, por sí mismas, no son las que facilitan el cambio social. En cambio, las personas, instituciones y organizaciones sí: al emplearlas, pueden convertirlas en herramientas para alcanzar objetivos de progreso, desarrollo, autonomía y emancipación. El reto para quienes queremos optar por una modernidad inclusiva es enfrentar el mito de que la tecnología es el fin, cuando en realidad es el medio. Divulgar esta concepción podría ayudar a cerrar muchas brechas 
desde los ámbitos más sencillos de las aplicaciones tecnológicas, puesto que se entendería el valor de la inversión y el conocimiento en tecnología para lograr metas superiores.

Una universidad no solo debe asumir procesos personales y humanizados para una población grande y diversa de personas que estudian y trabajan en ella. También debe hacer frente a la gestión de una enorme cantidad de información administrativa, financiera y propia de los procesos de docencia, investigación y acción social de las unidades académicas e investigativas. Por ello, considero necesario que las autoridades universitarias comprendan el impacto positivo que pueden tener las tecnologías de la información y comunicación (TIC) en el desempeño de las diferentes áreas de la universidad. Las inversiones para poder desarrollar los diversos servicios son altas, y el limitado presupuesto debe ser aprovechado para maximizar los beneficios y el impacto. Esto implica que se requiere un involucramiento de las autoridades en la toma de decisiones, especialmente en lo relacionado con la generación de políticas y la priorización de inversiones.

Las universidades del futuro, en especial aquellas que reciben recursos públicos en sus presupuestos, tienen la obligación de implementar los principios de gobierno abierto para asegurarle a la ciudadanía que sus aportes están siendo utilizados de la mejor forma posible, sin que esto signifique un menoscabo a los principios de la autonomía universitaria.

Desde nuestra filosofía, no importa cuánta tecnología se investigue, diseñe, implemente o adquiera, ni el nivel de desarrollo tecnológico que tenga una institución o cuánto invierte en sus TIC, si al final eso no se convierte en beneficios tangibles para las personas que integran la comunidad universitaria y no tiene un impacto positivo para la sociedad en general. Cada cosa que se hace usando TIC tiene que facilitar los procesos de quienes se relacionan con la universidad, potenciar el acceso al conocimiento, disminuir la brecha digital y aumentar la inclusión social. La investigación, por ejemplo, es uno de los pilares de las universidades que puede verse favorecido con las nuevas tecnologías. A fin de cuentas, nuestras sociedades -que están cada vez más orientadas hacia el conocimiento- deberán emprender caminos en los cuales las tecnologías formen cada vez más parte de la vida cotidiana de sus ciudadanos, pero para ello se debe pensar en mecanismos que lo promuevan, y las universidades cuentan con las plataformas necesarias y adecuadas para empezar o potenciar estos procesos.

Asimismo, la internacionalización es uno de los retos que más atañe a nuestras universidades latinoamericanas. Mientras que anteriormente la internacionalización se veía como un proceso de recibir cooperación desde instituciones o países más "desarrollados" hacia otros menos desarrollados, ahora sabemos que el conocimiento se genera mediante experiencias compartidas y desarrolladas por todos los entes participantes, de ahí que acciones de cooperación más horizontales se han venido generando a nivel general.

Formar parte de sas tendencias mundiales es un requisito de las universidades modernas, y América Latina debe insertarse en estas dinámicas con calidad y competencia. Es importante destacar que la promoción de la educación mediante los procesos de internacionalización siempre se da en un camino de dos vías: por una parte, se recibe un enorme acervo educativo y cultural del país con el cual nos relacionamos, pero también tenemos la oportunidad única de transmitir conocimientos propios, autóctonos y derivados de nuestra propia experiencia, como una manera de valorizar los saberes que en nuestros países se generan y se estudian. El conocimiento no debe concebirse en términos aislados, sino que debe entenderse que el enriquecimieto producto de las relaciones externas de una universidad siempre es mutuo.

¿Por qué deberíamos buscar y establecer convenios con instituciones extranjeras? No se trata únicamente de mejorar la proyección internacional y fortalecer el respaldo institucional, sino también para promover los contactos estratégicos, conseguir financiamiento, establecer 
compromisos, y honrar las responsabilidades y condiciones, así como la transparencia, en nuestras relaciones externas. $Y$ es importante mencionar que esto puede, y debe, incluir también las propias relaciones entre las instituciones académicas de nuestra región, puesto que aquí mismo en América Latina y el Caribe contamos con diversas necesidades de aprendizaje que entre nuestras mismas instituciones ya se han ido solventado. Es decir, el fortalecimiento puede ser intraregional, y esto no hará más que aumentar el valor agregado de las ofertas de formación que podamos tener para nuestras y nuestros estudiantes, quienes podrían tener la posibilidad de conocer de cerca lo que países hermanos estudian actualmente.

Finalmente, aspectos como la burocracia interna en las propias universidades, así como la crisis presupuestaria que afecta a nuestros países y la falta de financiamiento adecuado para los quehaceres académicos, son retos que nuestras instituciones de educación superior deben enfrentar de la mano con planes y estrategias pormenorizadas para que su existencia sea prolongada, sea reconocida como necesaria, y sea adecuada para responder con calidad y ética a las necesidades de cada uno de nuestros países.

\section{Conclusiones}

La autonomía de las universidades públicas latinoamericanas como legado de la Reforma de Córdoba es de un rango tan especial que no dudo en calificarla de un verdadero logro de la evolución política y cultural, rasgo que nos distingue de las otras regiones del mundo. Entre nosotros, aquí en Nuestra América, la autonomía universitaria es especial, es completa y por esto, cuenta la universidad pública con todas las facultades y poderes administrativos necesarios para llevar adelante el fin especial que legítimamente se le ha encomendado; que puede autodeterminarse, en el sentido de que está posibilitada para establecer sus planes, programas, presupuestos, organización interna y estructurar su gobierno propio. Tiene poder reglamentario (autónomo y de ejecución); puede autoestructurarse, repartir sus competencias dentro del ámbito interno del ente, desconcentrarse en lo jurídicamente posible y lícito, regular el servicio que prestan, y decidir libremente sobre su personal. Son estas las modalidades administrativa, política, organizativa y financiera de la autonomía que corresponde a las universidades públicas.

La autonomía universitaria tiene, como principal finalidad, procurar al ente todas las condiciones jurídicas necesarias para que lleve a cabo con independencia su misión de cultura y educación superiores. En este sentido, la universidad no es una simple instituciónón de enseñanza, pues a ella corresponde la función compleja, integrante de su naturaleza, de realizar y profundizar la investigación científica, cultivar las artes y las letras en su máxima expresión, analizar y criticar, con objetividad, conocimiento y racionalidad elevados, la realidad social, cultural, política y económica de su pueblo y el mundo, proponer soluciones a los grandes problemas y por ello en el caso de los países subdesarrollados, o poco desarrollados, como el nuestro, servir de impulsora a ideas y acciones para alcanzar el desarrollo en todos los niveles (espiritual, científico y material), contribuyendo con esa labor a la realización efectiva de los valores fundamentales de la identidad latinoamericana, que pueden resumirse, según se dijo en el voto que se acaba de citar, en los de la democracia, el Estado Social de Derecho, la dignidad esencial del ser humano y el "sistema de libertad", además de la paz y la justicia; en síntesis, para esos propósitos es creada, sin perjuicio de las especialidades o materias que se le asignen, y nada menos que eso se espera y exige de ella. La anterior conceptuación no persigue agotar la totalidad de los elementos, pero de su contenido esencialmente se deduce que la universidad, como centro de pensamiento libre, debe y tiene que 
estar exenta de presiones o medidas de cualquier naturaleza que tiendan a impedirle cumplir, o atenten contra ese, su gran cometido.

\section{Bibliografía}

Arocena, Rodrigo (2011), Los desafíos de la universidad pública en América Latina. Conferencia de Rector de la Universidad de la República de Uruguay en la Universidad de Chile. Santiago de Chile, julio. http://www.uchile.cl/seminario-educacion

Banco Mundial (1996), Prioridades y estrategias para la educación. Examen del Banco Mundial. Banco Internacional de Reconstrucción y Fomento. Washington, D. C.

Banco Mundial (2015), "Gasto en investigación y desarrollo (\% del PIB)". http://datos.bancomundial.org/indicador/GB.XPD.RSDV.GD.ZS

Baudrit, Luis (2011), Espíritu universitario y autonomía. Autonomía universitaria. Condición de un pueblo libre. Reflexiones 4-7.

Banco Mundial (2014), Conversatorio Autonomía universitaria costarricense en tiempo de cambio. Estado de la Nación, Universidad de Costa Rica, Instituto Tecnológico de Costa Rica y la Universidad Nacional.

Blanco, Patricia (2012), Sentencia reafirma libertad de cátedra. Oficina de Divulgación e información. Universidad de Costa Rica. https://www.ucr.ac.cr/noticias/2012/10/29/sentenciareafirma-libertad-de-catedra.html

Bobbio, Norberto (1992), Liberalismo y democracia. México: Brevarios del Fondo de Cultura Económica.

Braudel, Fernand (1980), La Historia y las Ciencias Sociales. Madrid: Alianza Editorial.

Brenes, Lizette (2014), Conversatorio "Autonomía universitaria costarricense en tiempo de cambio". Estado de la Nación, Universidad de Costa Rica, Instituto Tecnológico de Costa Rica y la Universidad Nacional.

Brunner, José (2010). Globalización de la educación superior: crítica de su figura ideológica. Revista Iberoamericana de Educación Superior 2. España.

Brunner, José (2001), Peligro y promesa: educación superior en América Latina. López Segrera, F. y A. Maldonado (Coords.) Educación Superior Latinoamericana y Organismos Internacionales. Un Análisis Crítico. Cali. UNESCO-Universidad de San Buenaventura, Cali, Colombia-Boston Collage. pp.

93-166. http://200.6.99.248/ bru487cl/files/PELIGRO\%20Y\%20PROMESA_es\%20EN\%20AL_2_.pdf

Brunner, José (1990), Educación Superior en América Latina: cambios y desafíos. Santiago: Fondo de Cultura Económica. 
Brunner, José y Elacqua, Gregory (2005), Factores que inciden en una educación efectiva: evidencia internacional http://www.educarchile.cl/ech/pro/app/detalle?ID=106885

Camacho, Daniel; Dobles, Ignacio; González, Mirta; Ramírez, Francisco; Rivas, Francisco y Wing, Isabel (1980), Defensa de la Autonomía Universitaria. Ponencias IV Congreso Universitario. Universidad de Costa Rica. pp. 4-10.

Cantard, Albor (2013), En defensa de la autonomía. Reunión Región Cono Sur de Unión de Universidades de América Latina y el Caribe (UDUAL). Universidad Nacional de La Plata. http://www.udual.org/revistauniversidades/doc55-2.html

Carta Magna (1988), "Carta Magna de las Universidades Europeas" http://firgoa.usc.es/drupal/files/CARTAMAGNA.pdf

Castells, Manuel (ed) (2006), La sociedad red: Una visión global. Madrid: Alianza Editorial.

Córdoba, Javier (2010), "Luis Baudrit: La policía no puede entrar al campus como si fuera tierra de nadie" Semanario Universidad. San José: Universidad de Costa Rica.

De Sousa, Boaventura (2005), La Universidad en el siglo XXI. Para una reforma democrática y emancipadora de la Universidad. México: Centro de Investigaciones Interdisciplinarias en Ciencias y Humanidades. Universidad Nacional Autónoma de México (UNAM).

Declaración de La Sorbona (1998), "Declaración de La Sorbona". Declaración conjunta para la armonización del diseño del Sistema de Educación Superior Europeo. París, 25 mayo. www.uhu.es/convergencia_europea/documentos/documentos-2006/documento-sorbona1998.pdf

Declaración de Bolonia (1999), Declaración de Bolonia. Espacio Europeo de Enseñanza Superior. Declaración conjunta de los ministros europeos de enseñanza. www.educacion.gob.es/boloniaensecundaria/img/Declaracion_Bolonia.pdf

Federación Universitaria de Córdoba (2008), “Manifiesto Liminar de la Reforma Universitaria. La juventud argentina de Córdoba a los hombres libres de Sud América." Cuadernos del Pensamiento Crítico Latinoamericano 5. Buenos Aires: Consejo Latinoamericano de Ciencias Sociales. (CLACSO).

Freire, Pablo (2008), Pedagogía del oprimido. Buenos Aires: Editorial Siglo Veintiuno.

Galdamés, Luis (1935), La autonomía universitaria. San José: Editorial Borrasé.

Ginés-José (2002), “El gobierno de las universidades: en búsqueda de una adecuada combinación de autonomía y eficiencia" Conferencias Internacionales. San José. VI Congreso Universitario. Universidad de Costa Rica. pp. 5-25.

Halperin, Tulio (2005), Historia contemporánea de América Latina. Madrid: Alianza Editorial, Libro de Bolsillo. 
Huaylupo, Juan (2016), "La institucionalidad y universidad pública ¿Un tributo individualista?" Diario Digital El País. Opinión. San José, 05 de abril. http://www.elpais.cr/2016/04/05/lainstitucionalidad-y-universidad-publica-un-tributo-individualista/

Huaylupo, Juan (2015), "Nacionalización de la institucionalidad pública: paradójica consecuencia liberal." Semanario Universidad. Edición № 2113. Opinión. Semana 25/11 al 1\%12 pg. 20. http://semanariouniversidad.ucr.cr/opinion/nacionalizacion-de-la-institucionalidad-publicaparadojica-consecuencia-liberal/

Huaylupo, Juan (2010), "La incomunicación social en la globalización de las técnicas comunicativas" Revista Ciencias Económicas. 28(2) pp. 447-462.

Huaylupo, Juan (2001), Las organizaciones y las ciudades en tiempos de privatización mundial del bienestar. Revista Reflexiones. 80(2). 103-114.

Huaylupo, Juan (1999), La política pública en un contexto de privatización de la política social del Estado. Revista Mexicana de Sociología. Instituto de Investigaciones Sociales. Universidad Nacional Autónoma de México (UNAM). 4(99) pp. 55-72. http://www.jstor.org/pss/3541191;

Huguet, Andrés (1992), Las tesis de Fukuyama sobre el fin de la historia. (Una crítica elemental). Ediciones Avances. Lima: www.huguet.tripood.com/fukuyama.htm

Jaramillo, Guillermo (2003), ¿Qué es epistemología? Revista Cinta de Moebio, 18.

Jensen, Henning (2013a), Informe de labores 2012-2013. Universidad de Costa Rica. http://www.rectoria.ucr.ac.cr/www/index.php/informe-de-labores-2012-2013

Jensen, Henning (2013b). "UCR suscribió Carta Magna Universitaria. Rector estrechó relaciones con universidades europeas y árabes." http://www.ucr.ac.cr/noticias/2013/10/25/ucr-suscribiocarta-magna-universitaria.html

Jensen, Henning (2012), “Entrevista a Henning Jensen: ¿Está desconectada la UCR de las necesidades del país?". Semanario El Financiero. San José, 10 junio.o http://www.elfinancierocr.com/economia-y-politica/Entrevista-Henning_Jensen-UCR-rectorInterrogatorio_0_140985963.html

López, Francisco (2008), Tendencias de la educación superior en el mundo y en América Latina y el Caribe. Avaliação. Revista da Avaliação da Educação Superior. Universidad de Sorocaba, Brasil, 13(2), pp. 267-291,

Lima, Luis (1994), "Prólogo" 75 Aniversario de la Reforma Universitaria. Argentina: Editorial de la Universidad Nacional de La Plata. Tomo I, pp. 18-25.

Lins, Gustavo y Escobar, Arturo (2006) "Las antropologías del mundo. Transformaciones de la disciplina a través de los sistemas del poder. Revista Universitas Humanística. Pontificia Universidad Javeriana. Bogotá. 61, pp. 15-49. 
Madrid, Raúl (2013), El derecho a la libertad de cátedra y el concepto de universidad. Ensayos y Crónicas. Revista Chilena de Derecho. 40(1), pp. 353-369.

Madrigal, Luis (2015), "UCR beca a más del $50 \%$ de sus estudiantes, casi el $90 \%$ de colegios públicos." http://www.elmundo.cr/ucr-beca-a-mas-del-50-de-sus-estudiantes-casi-el-90-decolegios-publicos/

Madriz, Allan (2016), "PLN no investigará cátedra de cultura islámica impartida en la UCR" elPeriódicocr. San José, 7 de noviembre. http://elperiodicocr.com/pln-no-investigara-catedra-decultura-islamica-impartida-en-la-ucr/

Maldonado, Carlos (2011), Complejidad, Ciencia y América Latina. Entrevista a Carlos Maldonado por Manuel Paz y Miño. Revista-E Latinoamericana de Filosofía Aplicada. Lima: filosofiaaplicadalatinoamericana. Blogpot.com/2011/2/complejidad-ciencia-y-america-latina.html

Manifiesto (2011), Autonomía universitaria: condición de un pueblo libre. Manifiesto de las universidades públicas de Costa Rica. Universidad de Costa Rica, Universidad Nacional, Instituto Tecnológico de Costa Rica y Universidad Estatal a Distancia. http://www.uned.ac.cr/conuniversitario/images/docs_cu/pronunciamientos/AUTONOMIAMANIFI ESTO.pdf

Mariátegui, José (1977). "La Reforma Universitaria. Ideología y Reivindicaciones". 7 Ensayos de interpretación de la realidad peruana. Obras Completas Tomo II. Lima. Biblioteca Amauta. Empresa Editora Amauta. pp. 122-161.

Monge, Carlos (1970), La Universidad Contemporánea. Análisis crítico, principios, metas y objetivos. San José. Publicaciones de la Universidad de Costa Rica. Serie Misceláneas № 140.

Morin, Edgar (1999), Los siete saberes necesarios para la educación del futuro. París. Organización de las Naciones Unidas para la Educación, la Ciencia y la Cultura (UNESCO). http://unesdoc.unesco.org/images/0011/001177/117740so.pdf

PAIRCA y CSUCA (2008), Propuesta: Armonización de los sistemas de educación superior. Programa de formación e investigación en integración regional centroamericano. www.csuca.org/index.php/centro-de-documentacion/estudios-de-armonizacion

Ramírez, René (Coordinador) (2010), Transformar la Universidad para Transformar la Sociedad. Ecuador. Secretaria Nacional de Planificación y Desarrollo (SENPLADES). Colección Debates № 1.

Ramonet, Ignacio; Brune, Francois; Mattelart, Armand et al. (1998), Pensamiento crítico vs. Pensamiento único. Madrid: Le Monde Diplomatique. Editorial Debate.

Rovira, Jorge (1990), Reflexiones sobre el futuro de la universidad de Costa Rica. (Una contribución al V Congreso Universitario). V Congreso Universidad de Costa Rica. Comisión Regionalización. Universidad de Costa Rica. 
Sánchez, José (Director) (1998), Pensamiento Crítico vs. Pensamiento Único. Madrid: Temas de Debate. Le Monde Diplomatique.

Sánchez, Luis (1961), La Universidad no es una isla. Perú: Ediciones Perú.

Siles, Zaida (2011), Consejo Universitario reafirma libertad de cátedra. Universidad de Costa Rica. http://www.cu.ucr.ac.cr/inicio/noticias/noticia/Articulo/consejo-universitario-reafirma-libertadde-catedra.html

Tatián, Diego; Vázquez, Guillermo (2017), La autonomía hacia el centenario de la Reforma de Córdoba. UDUAL. Cuadernos de Universidades. https://www.udual.org/principal/wpcontent/uploads/2017/11/La-autonom\%C3\%ADa-hacia-el-centenario-de-la-Reforma-Universitariaweb.pdf.

Tünnermann, Carlos (2011), La universidad del futuro. Nicaragua: HISPAMER.

UNESCO (2005), Hacia las sociedades del Conocimiento. París. Informe Mundial de la UNESCO. Organización de las Naciones Unidas para la Educación, la Ciencia y la Cultura. http://unesdoc.unesco.org/images/0014/001419/141908s.pdf

UNESCO (1998), La educación superior en el siglo XXI Visión y acción. Conferencia Mundial sobre la Educación Superior. Organización de las Naciones Unidas para la educación, la ciencia y la cultura (UNESCO). París. ED-98/CONF.202/5

UNESCO (1994), Declaración mundial sobre educación para todos. París. UNESCO para la Secretaría del Foro Consultivo Internacional sobre Educación para Todos. http://www.unesco.org/education/pdf/JOMTIE_S.PDF

Ugarte, Yoselyne (2015), UCR respetará libertad de cátedra de docentes y no limita su expresión de ningún modo, crhoy.com San José, 23 marzo.

Valadés, Diego (2014), Autonomía y constitución en América Latina. Villar, Alejandro y Ibarra, Antonio (Compiladores). La autonomía universitaria, una mirada latinoamericana. México: Unión de Universidades de América Latina y el Caribe (UDUAL), Universidad Autónoma de México (UNAM) y Universidad de Campiñas (UNICAMP). pp. 23-35.

Vargas, Jorge (2007), La democracia y las restricciones institucionales al desarrollo de Costa Rica. Claudio Ansorena (Editor) Restricciones Institucionales al desarrollo de Costa Rica. San José.

Vargas, Mario (2009), Breve discurso sobre la cultura. Discurso a la concesión del Doctorado en Honoris Causa por la Universidad de Granada. España. http://www.ugr.es/ proto/documentos/DISCURSO\%20SOBRE\%20LA\%20CULTURA.\%20GRANADA. pdf 